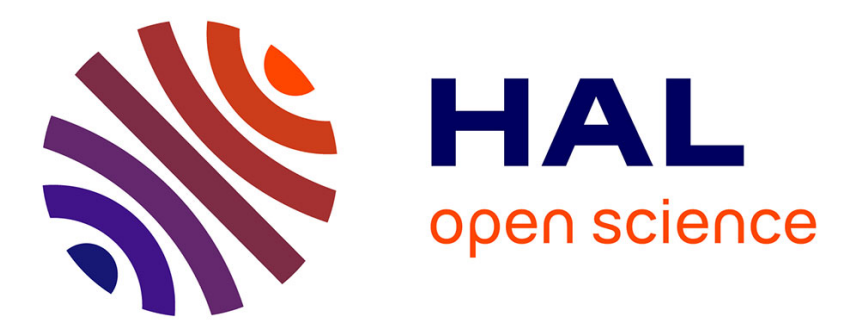

\title{
Observation of zero-sound at atomic wave-vectors in a monolayer of liquid $3 \mathrm{He}$
}

Henri Godfrin, Matthias Meschke, Hans-J. Lauter, Helga M. Böhm, Eckhard

Krotscheck, Martin Panholzer

\section{- To cite this version:}

Henri Godfrin, Matthias Meschke, Hans-J. Lauter, Helga M. Böhm, Eckhard Krotscheck, et al.. Observation of zero-sound at atomic wave-vectors in a monolayer of liquid 3He. Journal of Low Temperature Physics, 2010, 158, pp.147. 10.1007/s10909-009-9952-5 . hal-00921371

\section{HAL Id: hal-00921371 \\ https://hal.science/hal-00921371}

Submitted on 20 Dec 2013

HAL is a multi-disciplinary open access archive for the deposit and dissemination of scientific research documents, whether they are published or not. The documents may come from teaching and research institutions in France or abroad, or from public or private research centers.
L'archive ouverte pluridisciplinaire HAL, est destinée au dépôt et à la diffusion de documents scientifiques de niveau recherche, publiés ou non, émanant des établissements d'enseignement et de recherche français ou étrangers, des laboratoires publics ou privés. 


\title{
Observation of zero-sound at atomic wave-vectors in a monolayer of liquid ${ }^{3} \mathrm{He}$
}

\author{
H. Godfrin • M. Meschke • H.-J. Lauter •
}

H.M. Böhm · E. Krotscheck · M. Panholzer

Received: date / Accepted: date

\begin{abstract}
The elementary excitations of a strongly interacting two-dimensional Fermi liquid have been investigated by inelastic neutron scattering in an experimental model system: a monolayer of liquid ${ }^{3} \mathrm{He}$ adsorbed on graphite preplated by a monolayer of solid ${ }^{4} \mathrm{He}$. We observed for the first time the particle-hole excitations characterizing the Fermi liquid state of two-dimensional liquid ${ }^{3} \mathrm{He}$, and we were also able to identify the highly interesting zero-sound collective mode above a particle-hole band. Contrarily to bulk ${ }^{3} \mathrm{He}$, at low wave-vectors this mode lies very close to the particle-hole band. At intermediate wave-vectors, the collective mode enters the particle-hole band, where it is strongly broadened by Landau damping. At high wave-vectors, where the Landau theory is not applicable, the zero-sound collective mode reappears beyond the particlehole band as a well defined excitation, with a dispersion relation quite similar to that of superfluid ${ }^{4} \mathrm{He}$. This spectacular effect is observed for the first time in a Fermi liquid (including plasmons excitations in electronic systems).
\end{abstract}

Keywords Quantum fluids $\cdot{ }^{3} \mathrm{He} \cdot$ Fermi Liquid · excitations · inelastic neutron scattering

PACS 67.10.-j $\cdot 67.30 . \mathrm{E}-\cdot 67.30 . \mathrm{em} \cdot 71.27 .+\mathrm{a}$

H. Godfrin

Institut Néel, CNRS et Université Joseph Fourier, BP 166, F-38042 Grenoble Cedex 9, France Tel.: +33476889063

Fax: +33456387087

E-mail: henri.godfrin@grenoble.cnrs.fr

M. Meschke

Institut Néel, CNRS et Université Joseph Fourier, BP 166, F-38042 Grenoble Cedex 9, France Present address: Low Temperature Laboratory, Helsinki University of Technology P.O. Box 3500, FIN-02015 TKK, Finland

H.-J. Lauter

Institut Laue-Langevin, BP 156, 38042 Grenoble Cedex 9, France

H.M. Böhm · E. Krotscheck · M. Panholzer

Institute for Theoretical Physics, Johannes Kepler University, Austria 


\section{Introduction}

Our experimental research on liquid ${ }^{3} \mathrm{He}$ in two and three dimensions has been strongly motivated by the extraordinary lectures and courses on Quantum Fluids given by Philippe Nozières [1] in Grenoble for many years. The classical books of Pines and Nozières [2], Fetter and Walecka [3] and Wilks [4], the original papers by Landau and a remarkable article published by D. Pines in Physics Today [5], which emphasizes the important open questions, have also inspired considerably our work. The aim of the experiments described here is to improve our current understanding of the fundamental quantum properties of Fermi liquids, and in particular their dynamics at elevated wave-vectors. For this purpose, as we shall see below, the determination of the dynamic structure factor of liquid ${ }^{3} \mathrm{He}$ at very low temperatures, essentially near the absolute zero of temperature, plays an important role. This function of the energy and momentum contains all the relevant information on the elementary excitations of the system under investigation. Neutron scattering is a privileged tool for such studies, and this technique has been extensively used for the observation of phonons, magnons and other types of excitations in condensed matter. In quantum fluids, the direct observation of a phonon-maxon-roton dispersion relation in excellent agreement with the theoretical predictions of Landau constitutes one of the major results for the physics of bosonic systems. In the case of fermions, like bulk liquid ${ }^{3} \mathrm{He}$, neutron scattering has allowed the observation of particle-hole excitations as well as the zero-sound collective mode, predicted by Landau's theory of Fermi liquids $[2,6]$.

\subsection{Excitations in Fermi liquids}

The simplest excitation of a Femi liquid at zero temperature is the creation of a particlehole pair. This is achieved by promoting a particle from the Fermi sea (Fermi sphere in three dimensions) to another state, necessarily located outside the Fermi sphere in order to satisfy the requirements of the Pauli principle. Simple considerations show that the possible excitations are confined to a particular region of the energy-momentum space, "the particle-hole band (PHB)" (Figure 1). For a given momentum, a minimum and maximum energy determine the two boundaries of the PHB. For a non-interacting system (Fermi gas), these boundaries can be calculated using the energy of a free ${ }^{3} \mathrm{He}$ atom, $E=\hbar^{2} k^{2} / 2 m$, where $m$ is the mass of the ${ }^{3}$ He atom. In a Fermi fluid, the Landau theory postulates the existence of "quasiparticles" as the elementary excitations. It assigns to these quasiparticles an effective mass $m^{*}$ which is a measure for the density of states at the Fermi surface and is, therefore, observable by heat capacity experiments. In ${ }^{3} \mathrm{He}, m^{*} / m$ is substantially larger than one, and increases with the interactions (pressure). Therefore, the PHB could be expected to shift to energies well below those calculated with the bare mass of a ${ }^{3} \mathrm{He}$ atom, for which $m^{*}=m$. As we shall see below, such a crude extrapolation of the Landau theory far ouside its validity domain does not agree with the data, and a more sophisticated theory will be used to describe the physics at high energies and wave-vectors.

In bulk liquid ${ }^{3} \mathrm{He}$, the existence of two collective modes [2] has been predicted by Landau. The first one is described as a collective oscillation of the Fermi sphere, while the second one consists of spin waves associated to the spin degree of freedom of the system. The zero-sound mode is "collisionless": contrarily to ordinary sound, its frequency is higher than the collision rate. It has been detected long ago by ultrasonic 


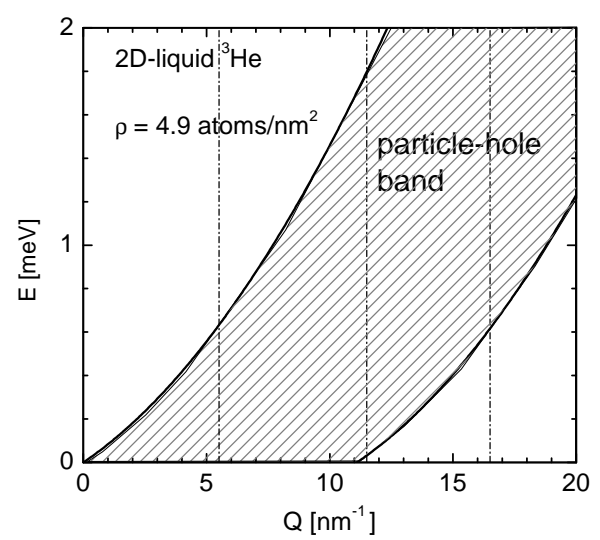

Fig. 1 The particle-hole excitations band, characteristic of a Fermi liquid, is shown for 2Dliquid ${ }^{3} \mathrm{He}$ for a density of 4.9 atoms $/ \mathrm{nm}^{2}$ (shaded region). At zero energy, the band extends from zero momentum to $2 k_{F}$, where the Fermi wave-vector is $k_{F}=5.55 \mathrm{~nm}^{-1}$. The energy is calculated using the bare mass of the ${ }^{3} \mathrm{He}$ atoms. The Fermi energy is $E_{F}=0.213 \mathrm{meV}$ for this areal density. The presence of collective modes is investigated in this energy-momentum space; typical results are given in the next figures along the constant $Q$ lines shown here.

techniques, at very low wave-vectors, at low enough temperatures. It has also been directly investigated by neutron scattering, from rather low up to very high wavevectors (on the order of $\mathrm{k}_{F}$ ). In this region of the energy-momentum space, zero-sound is seen initially as a well defined mode with a linear dispersion relation, lying well above the PHB until, for large wave-vectors, it displays a negative curvature and enters the $\mathrm{PHB}$. In this new regime, zero-sound can decay in incoherent $\mathrm{PH}$ excitations, and a substantial broadening of the collective mode, named "Landau damping" is observed: the collective mode disappears in the $\mathrm{PH}$ continuum $[2,6]$. The same physics is observed in electronic systems: the collective mode, named "plasmon" in this context [2], displays at long wavelengths a gap due to the charged nature of this fermionic system, but at elevated wave-vectors, the plasmon dispersion relation behaves like the zero-sound mode of liquid ${ }^{3} \mathrm{He}$ : it hits the PHB and vanishes.

Two-dimensional liquid ${ }^{3} \mathrm{He}$ is a particularly interesting model system for correlated fermions. It has been extensively investigated by thermodynamic techniques in many low temperature laboratories (Seattle, Grenoble, London, Bell Laboratories, Amherst, etc.) [7-14]. The results clearly display the main features of Fermi liquids: linear heat capacity and constant magnetization as a function of temperature. This system presents, in addition to the reduced dimensionality, the advantage that a very large range of densities is experimentally accessible. There is no critical point, and therefore one can study densities from essentially zero (Fermi gas) to very large densities, near solidification. Heat capacity and magnetization measurements have shown that the effective mass varies as a function of density from $m^{*}=m$ to very large values, leading to interesting physics of two-dimensional systems, directly related to heavy Fermions, High $T_{c}$ superconductors, and quantum critical points, for example. 
However, nothing was known about the PHB and the collective modes of twodimensional ${ }^{3} \mathrm{He}$. In this conference, we present some results of the first investigation of the elementary excitations of two-dimensional liquid ${ }^{3} \mathrm{He}$. In the next section we provide details on the experimental set-up, and in the following sections several examples of the experimental results will be given. We conclude with a discussion on the implications, showing that new physics emerges at very high wave-vectors, which is of general interest for Fermi systems.

\section{Experimental details}

2.1 Two-dimensional liquid ${ }^{3} \mathrm{He}$ films

The two-dimensional liquid ${ }^{3} \mathrm{He}$ sample is produced by the physical adsorption of ${ }^{3} \mathrm{He}$ gas on a solid substrate. At low temperatures, well below $1 \mathrm{~K}$, the fluid motion is confined in the plane. The solid substrate used in the present work is a high quality exfoliated graphite (ZYX-UCAR) [9]. This type of exfoliated graphite has a small specific area (about $2 \mathrm{~m}^{2} / \mathrm{g}$ ), but, as seen later, this is not a major inconvenience. It presents many advantages, however: it has very large atomically flat platelets, and a small mosaic spread. Because of these characteristic, we have intensively used this sample in former works, in particular for diffraction studies of the solid phases of adsorbed ${ }^{3} \mathrm{He}$ which are still the reference in the field $[9,10]$. In addition, we used a well known technique of surface science, which consists of preplating the graphite substrate by a dense monolayer of ${ }^{4} \mathrm{He}$. This solid layer has a triangular structure, with a small lattice parameter $[9,10]$. Its adsorption potential for the next layer is weaker than that of bare graphite, which does not favor the formation of commensurate phases. Therefore, the second layer fluid is stable in a larger range of densities than the first layer. It should be pointed out that a substantial fraction of the adsorbed atoms is localized by heterogeneities (defects) of the bare graphite; the ${ }^{4} \mathrm{He}$ pleplating, in addition, smoothes out the defects of the graphite substrate, rendering the second layer fluid almost ideal.

The amount of ${ }^{4} \mathrm{He}$ and ${ }^{3} \mathrm{He}$ gas adsorbed onto the sample are carefully controlled by an adsorption isotherm and gas handling system [9]. The determination of the amount of ${ }^{4} \mathrm{He}$ needed to form a dense monolayer as first layer preplating, and the areal density of the low density fluid ${ }^{3} \mathrm{He}$ second layer is based on our previous neutron scattering experiments. We shall not discuss this point here in further detail. The essential point is that in this experiment the second layer is a uniform, pure ${ }^{3} \mathrm{He}$, atomically-thick liquid layer of known areal density. The data presented here correspond to a two-dimensional ${ }^{3} \mathrm{He}$ fluid layer of areal density 4.9 atoms $/ \mathrm{nm}^{2}$. The interparticle distance at this coverage correspods to that of bulk liquid ${ }^{3} \mathrm{He}$ at low pressures, and the effective mass of the $2 \mathrm{D}$ system is $m^{*} \approx 3.5 \mathrm{~m}$, also similar to the corresponding results for the bulk system [11].

\subsection{Experimental cell and cryogenics}

The graphite substrate must be cooled down to very low temperatures. For this reason, the graphite sample has been thermally bonded to copper foils, which are welded to a copper post attached to the copper cap of the experimental cell. The latter is 
thermally connected to the mixing chamber of a dilution refrigerator specially designed for neutron scattering studies: there is no liquid helium or nitrogen in the neutron path, and aluminum is used for the screens and vacuum chambers traversed by the neutrons. The cell body is made out of aluminum. A filling capillary connects the cell to the sample gas handling system. The experimental cell reaches temperatures well below $100 \mathrm{mK}$, which is a convenient range for investigating excitations of order $0.1 \mathrm{meV}$.

\subsection{The neutron spectrometer}

The inelastic neutron scattering experiments were performed at the Institut Laue Langevin (ILL) on the instrument IN6. This time-of-flight neutron spectrometer has detectors in the angular range 11.9-113 degrees. Taking into account the incident neutrons wavelength of $0.512 \mathrm{~nm}$, this corresponds to an accessible momentum transfer (Q) range $2.54<Q<20.46 \mathrm{~nm}^{-1}$ for elastically scattered neutrons ("elastic $Q$ "), and a somewhat smaller range at finite energy transfer (a few meV in the present work, limited by the incident neutron energy). The energy resolution is of the order of $0.1 \mathrm{meV}$. The sample c-axis is oriented vertically, i.e. perpendicular to the scattering plane.

Measurements are first done on a vanadium sample in order to calibrate the detectors. An empty cell scan is made, to be used as background and reference. Spectra are recorded for different coverages, at different temperatures. Scans at high temperatures, where the ${ }^{3} \mathrm{He}$ sample is in the gas phase, have also been measured in order to check the background and absorption corrections. Due to the small amount of adsorbed ${ }^{3} \mathrm{He}$ in the sample, the data acquisition takes about 20 hours for each spectrum in order to have significant statistics. In addition, the very large absorption cross section of the ${ }^{3}$ He nucleus leads to a substantial absorption. This limits the dimensions of the sample, thus reducing the available signal, and makes the analysis (background subtraction) particularly difficult.

\section{Results}

In the following, we present the results obtained for different momentum transfers. We first discuss the "low wave-vector" region, where experiments in the bulk liquid have shown the existence of a well defined zero-sound mode $[6,15-17]$ well above the PHB (particle-hole band).

We show in Figure 2 the spectrum obtained on our two-dimensional Fermi liquid for a momentum transfer $Q=5.5 \mathrm{~nm}^{-1}$. At small energies (less than $0.3 \mathrm{meV}$, the subtraction of the large elastic signal of the cell (background) hinders a precise determination of the spectrum. Above this value, the signal can be conveniently exploited. The most striking result is the absence of the collective mode at energies of about $1 \mathrm{meV}$, as observed in the bulk liquid at different pressures $[16-18,6]$. The second observation is the presence of a large intensity (for such small $Q$ values) mode, just above the PHB (Fig. 2). This is the two-dimensional zero-sound collective mode, observed for the first time, as clearly identified by our complete set of data and the corresponding theoretical results, which cover the whole energy-momentum plane [19].

In Figure 3 we show the spectrum obtained for a momentum transfer $Q=11.5 \mathrm{~nm}^{-1}$. It corresponds, according to Fig. 3, to the region dominated by the PHB. As expected, the signal is weak. However, one can clearly observe a broad mode, which corresponds 


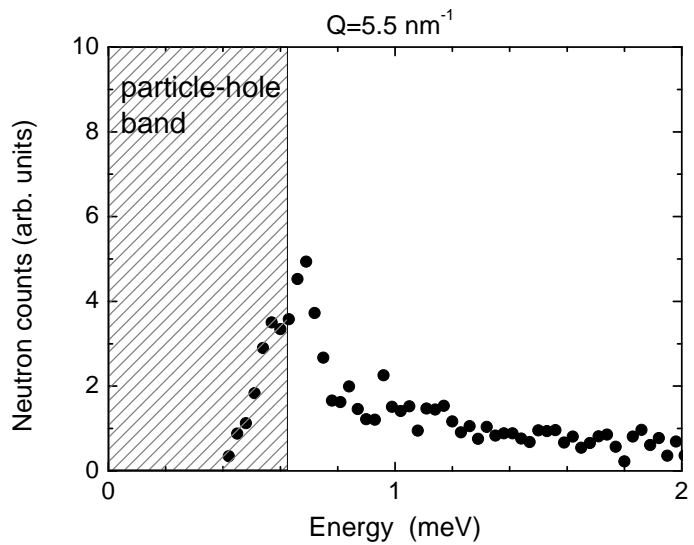

Fig. 2 Inelastic neutron scattering spectrum at a wave-vector of $5.5 \mathrm{~nm}^{-1}$. Note the mode above the particle-hole band.

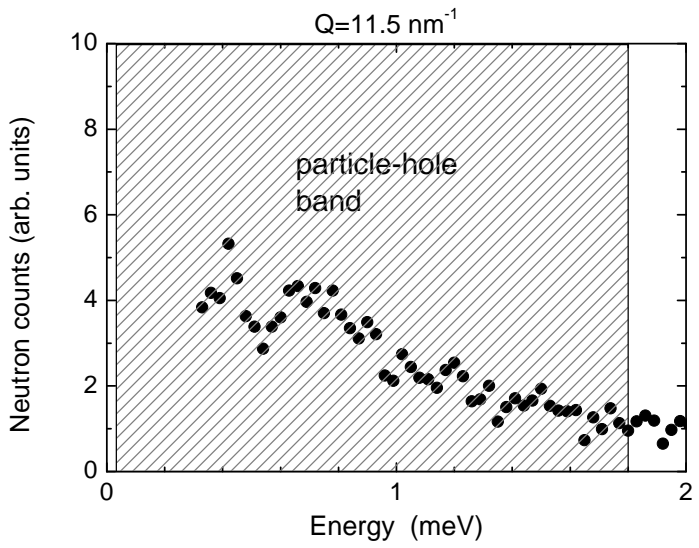

Fig. 3 Inelastic neutron scattering spectrum at a wave-vector of $11.5 \mathrm{~nm}^{-1}$. Note the broad mode inside the particle-hole band.

to the damping of zero-sound within the PHB. Note that in the present article we have considered the PHB for $m^{*}=m$.

The behavior at higher wave-vectors is presented in Figure 4, where the momentum transfer is $Q=16.5 \mathrm{~nm}^{-1}$. The striking features are now the appearence of a large intensity peak below the PHB, again calculated for $m^{*}=m$. We have been able to demonstrate [19] that this intensity corresponds to the collective zero-sound mode reappearing beyond the $\mathrm{PH}$ continuum, at elevated wave-vectors.

The experimental data show therefore many important and novel features. At low wave-vectors, the presence of a collective mode, which is in the 2D system substantially lower than in the bulk. This effect, which results from the smaller restoring forces 


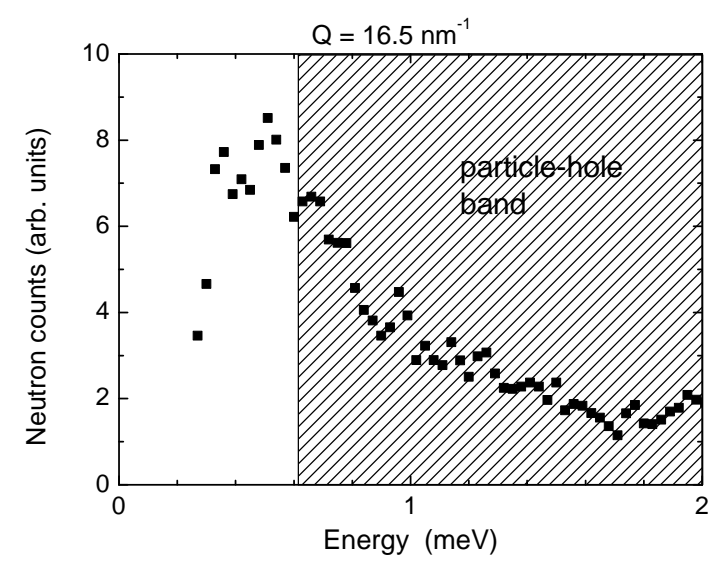

Fig. 4 Inelastic neutron scattering spectrum at a wave-vector of $16.5 \mathrm{~nm}^{-1}$. Note the mode below the particle-hole band.

associated to the reduced number of neighboring atoms, is well known for plasmons in electronic systems, and it has also been shown for $2 \mathrm{D}{ }^{3} \mathrm{He}$ by theoretical calculations [20].

The most spectacular observation is the presence of a high energy collective mode essentially identical to the phonon-roton curve of ${ }^{4} \mathrm{He}$, damped in the region of coexistence with the incoherent particle-hole excitations, but well defined outside this range, even for atomic-like wave-vectors, substantially larger than $k_{F}$.

The high energy collective mode of a system of correlated fermions, or "zero-sound", appears therefore to be largely independent on the quantum statistics. This might be quite surprising, if we keep in mind the original Landau derivation of this collective mode as an oscillation of the Fermi sphere. However, for the elevated wave-vectors and energies of the excitations considered here, we are very far from the conditions of validity of the Landau theory. A more appropriate view is that proposed by D. Pines and coworkers [21], where the similarities between correlated bosonic and fermionic systems are emphasized.

A major theoretical breakthrough was achieved by the theoretical component of this collaboration. The theory builds upon exactly the notion that the physical mechanisms are, in correlated bosonic and fermionic systems, basically the same and, hence, generalizes the time-honored theory of pair fluctuations to fermions by including twoparticle excitations. In a companion article [24], a descrition of the theory is given, as well as a comparison with some of our experimental data. The key result of this joint experimental and theoretical effort is a detailed picture of a model Fermi liquid well beyond the Landau validity domain [19].

\section{Conclusions}

The elementary excitations of a strongly interacting 2D Fermi Liquid have been investigated by neutron inelastic scattering in a model system: liquid ${ }^{3} \mathrm{He}$ adsorbed on 
graphite. Our first observation of the zero-sound collective mode in $2 \mathrm{D}-{ }^{3}$ He shows a good agreement with the recent pair-excitation theory developed by Krotscheck and coworkers, even at very large, i.e. atomic, wave-vectors. Crossing of the collective mode (zero-sound, or plasmon) through the particle-hole band is observed for the first time in a Fermi many-body system. It corresponds to the well known phonon-maxon-roton dispersion relation of ${ }^{4} \mathrm{He}$. The origin of the zero-sound collective mode is clearly due to the effect of strong correlations, which make this mode largely independent on the quantum statistics. Its damping in the $\mathrm{p}-\mathrm{h}$ band provides valuable information on the interactions.

Acknowledgements We acknowledge the support of the Partenariat Hubert Curien "Amadeus" (French-Austrian collaboration). The Austrian team also acknowledges support from the Austrian Science Fund FWF under project P21264.

\section{References}

1. P. Nozières, Liquides et Solides Quantiques, series of lectures given in Grenoble (1983), unpublished.

2. D. Pines, P. Nozières, The Theory of Quantum Liquids, Benjamin, New York (1966)

3. A.L. Fetter, J.D. Walecka, Quantum Theory of Many Particle Systems, McGraw-Hill, New York (1971)

4. J. Wilks, Liquid and Solid Helium, Clarendon Press, Oxford (1967)

5. D. Pines, Physics Today 34, 106 (1981)

6. H.R. Glyde, Excitations in Liquid and Solid Helium, Clarendon Press, Oxford (1994)

7. S.W. van Sciver and O.E. Vilches, Phys. Rev. B 18, 285 (1978)

8. D.S. Greywall, Phys. Rev. B 41, 1842 (1990)

9. H. Godfrin and H.J. Lauter, Progress in Low Temp. Physics, Vol.XIV, Chapter 4, p.213-320, ed. W.P. Halperin, Elsevier Science B.V., Amsterdam (1995)

10. H. Godfrin and R.E. Rapp, Advances in Physics 44, 113 (1995)

11. K.D. Morhard, C. Bäuerle, J. Bossy, Y.M. Bunkov, S.N. Fisher, H. Godfrin, Phys. Rev. B 53, $2658(1996)$

12. A. Casey, H. Patel, J. Nyéki, B.P. Cowan and J. Saunders, Phys. Rev. Lett. 90, 115301 (2003)

13. E.R. Dobbs, Helium Three, Oxford University Press (2001)

14. M. Neumann, J. Nyéki, B.P. Cowan and J. Saunders, Science 317, 1356 (2007)

15. R. Scherm, W.G. Stirling, A.D.B. Woods, R.A. Cowley and G.J. Coombs, J. Phys. C: Solid State Phys. 7, L341 (1974)

16. K. Sköld, C.A. Pelizzari, R. Kleb and G.E. Ostrowski, Phys. Rev. Lett. 37, 842 (1976)

17. R. Scherm, K. Glucksberger, B. Fak, K. Sköld, A.J. Dianoux, H. Godfrin and W.G. Stirling, Phys. Rev. Lett. 59, 217 (1987)

18. H.R. Glyde, B. Fak, N.H. van Dijk, H. Godfrin, K. Glucksberger and R. Scherm, Phys. Rev. B 61, 1421 (2000)

19. H. Godfrin, M. Meschke, H.J. Lauter, H.M. Böhm, E. Krotscheck and M. Panholzer, to be published

20. E. S. Hernandez and M. M. Calbi, J. Low Temp. Phys. 120, 1 (2000)

21. C.H. Aldrich and D. Pines, J. Low Temp. Phys. 32, 689 (1978)

22. H.W. Jackson, Phys. Rev. 185, 186 (1969)

23. C.C. Chang and C.E. Campbell, Phys. Rev. B 13, 3997 (1976)

24. H.M. Böhm, E. Krotscheck, M. Panholzer, H. Godfrin, H.J. Lauter and M. Meschke (these Proceedings) 